\title{
Monte Carlo generators for hadron physics: updates on PHOKHARA and EKHARA generators ${ }^{\star}$
}

\author{
Henryk Czyż ${ }^{1}$, Patrycja Kisza ${ }^{1}$, and Szymon Tracz ${ }^{1}$ \\ ${ }^{1}$ Institute of Physics, University of Silesia, PL-40007 Katowice, Poland.
}

\begin{abstract}
A short review of current upgrades of the PHOKHARA and EKHARA generators is presented together with a report on the work in progress. The upgrades are based on a newly constructed model of the $\chi_{c_{i}}-\gamma^{*}-\gamma^{*}$ and $\chi_{c_{i}}-J / \psi^{*}-\gamma^{*}$ form factors. Within this model predictions were made for the electronic widths of $\chi_{c_{1}}$ and $\chi_{c_{2}}$ and, based on the event generators results, cross sections $e^{+} e^{-} \rightarrow \chi_{c_{1}}\left(\rightarrow J / \psi\left(\rightarrow \mu^{+} \mu^{-}\right) \gamma\right)$, $e^{+} e^{-} \rightarrow e^{+} e^{-} \chi_{c_{1}}$ and $e^{+} e^{-} \rightarrow e^{+} e^{-} \chi_{c_{1}}\left(\rightarrow J / \psi\left(\rightarrow \mu^{+} \mu^{-}\right) \gamma\right)$.
\end{abstract}

\section{Introduction}

The event generators PHOKHARA and EKHARA were developed to help measurements of the $e^{+} e^{-} \rightarrow$ hadrons + photons and $e^{+} e^{-} \rightarrow e^{+} e^{-}+$hadrons cross sections. From their first publications $[1,2]$ the groups developing the codes put an effort to guarantee technical precision of the simulations at the level of $0.01 \%$. In the same time models of hadron-photon interactions were developed and radiative corrections calculated and implemented into the event generator PHOKHARA to guarantee the physical accuracy at the level required by the experimental groups. Till now the radiative corrections were not implemented into the distributed version of the generator EKHARA, even if they were calculated. Problems with the low efficiency of the code for the processes $e^{+} e^{-} \rightarrow e^{+} e^{-}+$hadrons + photons where not yet solved even if the works towards the solution are well advanced.

For the processes simulated by the event generator PHOKHARA, the radiative corrections to the initial state radiation (ISR) were calculated in [3] and implemented in $[1,4]$. The final state radiation (FSR) requires not only calculation of the radiative corrections, but also modeling of the hadron-photon interactions. This was studied only for the most important hadronic final states: charged pion and kaon pair production [5-8] and proton-anti-proton pair production [9]. The latter mode was at first implemented with ISR corrections only [10]. For other hadronic final states: three- [11] and four- [12, 13] pion modes and lambda pair production with their subsequent decays [14], only ISR corrections are implemented.

The $e^{+} e^{-} \rightarrow \mu^{+} \mu^{-} \gamma$ reaction is a separate subject of the studies as the final state does not involve hadrons. It serves as one of the luminosity monitoring tools. For this process the radiative corrections were gradually added [4,

\footnotetext{
^Work supported in part by the Polish National Science Centre, gran number DEC-2012/07/B/ST2/03867 and German Research Foundation DFG under Contract No. Collaborative Research Center CRC-1044.
}

7] and finally the complete corrections were calculated and implemented into the event generator in [15].

A special care was devoted to the treatment of narrow resonances [16], when modeling the radiative return processes [8]. New physical and technical problems arise in the vicinity of narrow resonances, which were solved in the event generator implementations. Lately, in [17], a possibility of simulation of the hadronic processes in a scan mode was also added. In this mode only ISR corrections are implemented with the exception of the protonanti-proton pair production, where the Coulomb factor models approximately FSR corrections.

The first version of the EKHARA generator [2] was developed for the background studies at KLOE for the pion form factor measurements. Only later it was upgraded for studies of the $\gamma^{*}-\gamma^{*}$ processes starting with the reaction $e^{+} e^{-} \rightarrow e^{+} e^{-} \pi^{0}[18]$ and adding subsequently [19] better modeling of the $\pi-\gamma^{*}-\gamma^{*}$ form factor and possibility of the generation of the reactions $e^{+} e^{-} \rightarrow$ $e^{+} e^{-} \eta\left(\eta^{\prime}\right)$. In [19] also a new model of the $\eta\left(\eta^{\prime}\right)-\gamma^{*}-\gamma^{*}$ form factors was developed to give predictions in agreement with experimental data.

In this proceedings we report on the latest progress on the upgrades of both event generators EKHARA and PHOKHARA. Results of the studies [20] on a direct production of $\chi_{c_{1}}(1 P)$ and and $\chi_{c_{2}}(1 P), J^{++}, J=1,2$ charmonia in electron-positron annihilation are reported in Section 2. In Section 3 we report on the studies [21] on the prospects of the detailed investigations of the $\chi_{c_{i}}, i=$ $0,1,2$ properties at BELLE2, using measurements of the reactions $e^{+} e^{-} \rightarrow e^{+} e^{-} \chi_{c_{i}}$ and $e^{+} e^{-} \rightarrow e^{+} e^{-} \chi_{c_{i}}(\rightarrow J / \psi(\rightarrow$ $\left.\mu^{+} \mu^{-}\right) \gamma$ ). In Section 4 we give a short summary and sketch the near future developments in both event generators. 
Table 1. Predictions of the electronic widths of the $\chi_{c_{1}}$ and $\chi_{c_{2}}$ charmonia within recently published models.

\begin{tabular}{|c|c|c|c|c|}
\hline & {$[20]$} & {$[27]$} & {$[26]$} & {$[28]$} \\
\hline$\Gamma\left(\chi_{c_{1}} \rightarrow e^{+} e^{-}\right)[\mathrm{eV}]$ & 0.43 & 0.046 & 0.367 & 0.1 \\
$\Gamma\left(\chi_{c_{2}} \rightarrow e^{+} e^{-}\right)[\mathrm{eV}]$ & 4.25 & 0.037 & 0.137 & - \\
\hline
\end{tabular}

\section{PHOKHARA generator: direct $\chi_{c_{1}}$ and $\chi_{c_{2}}$ production in $e^{+} e^{-}$annihilation}

The production of the quarkonium states with even charge conjugation in $e^{+} e^{-}$annihilation is suppressed, as two photon exchange is necessary to produce them. Yet, with the luminosity of the nowadays colliders, the loop induced processes, leading to direct production of such states are within experimental reach. This possibility was investigated in detail in [20]. The best place to search for such processes is the BES-III experiment even if in the original proposal [22] this possibility was not investigated. The reaction $e^{+} e^{-} \rightarrow \chi_{c_{i}}\left(\rightarrow J / \psi\left(\rightarrow \mu^{+} \mu^{-}\right) \gamma\right), i=1,2$ is especially suited for such a measurement as the muons and photon are well identified within the BES-III detector. The $\chi_{c_{0}}$ production rate is proportional to the electron mass and thus beyond reach of any near future experiment. For any realistic prediction of the cross section a model of the amplitudes $\chi_{c_{i}} \rightarrow J / \psi^{*} \gamma^{*}, \chi_{c_{i}} \rightarrow \gamma^{*} \gamma^{*}$ and $\psi(2 S) \rightarrow \chi_{c_{i}}^{*} \gamma^{*}$ is needed with parameters describing well the observed decay widths $\Gamma\left(\chi_{c_{i}} \rightarrow J / \psi \gamma\right), \Gamma\left(\chi_{c_{i}} \rightarrow \gamma \gamma\right)$ and $\Gamma\left(\psi(2 S) \rightarrow \chi_{c_{i}} \gamma\right)$. Several models were built to meet this requirement [20, 23-28]. All of them are able to give predictions for these widths in agreement with the experimental data [29]. Yet, when using these models to predict the electronic widths of the $\chi_{c_{1}}$ and $\chi_{c_{2}}$ charmonia the models give predictions, which vary within one order of magnitude. The situation involving only recent papers is summarised in Table 1. The reason for these differences is clear: the predictions of the electronic widths involve the calculation of loop integrals and thus the shape of the form factors is very much important. While the widths, discussed above, fix only couplings at some well defined scales. It was shown also in [20] that the phases between different contributions are crucial for the size of the predicted electronic widths. The phases come from QED interactions and thus are almost fully predicted. Moreover the interferences give sizable contributions both to the electronic widths and the cross sections.

The non-reducible background for the $\chi_{c_{1}}$ and $\chi_{c_{2}}$ production in the reactions $e^{+} e^{-} \rightarrow \chi_{c_{i}}(\rightarrow J / \psi(\rightarrow$ $\left.\left.\mu^{+} \mu^{-}\right) \gamma\right), i=1,2$ is the $J / \psi$ production in radiative return with its subsequent decay to $\mu^{+} \mu^{-}$. This was discussed in [20], where it was shown that within the model developed there the main effect comes from the interference between the background and signal diagrams. We show it in Fig. 1 for $\chi_{c_{1}}$ and in Fig. 2 for $\chi_{c_{2}}$. We assume here a beam resolution of $1 \mathrm{MeV}$ for each beam and Gaussian distribution of energies within a beam. For $\chi_{c_{1}}$ there is also visible, but small, contribution coming from $Z^{0}$ exchange. This result

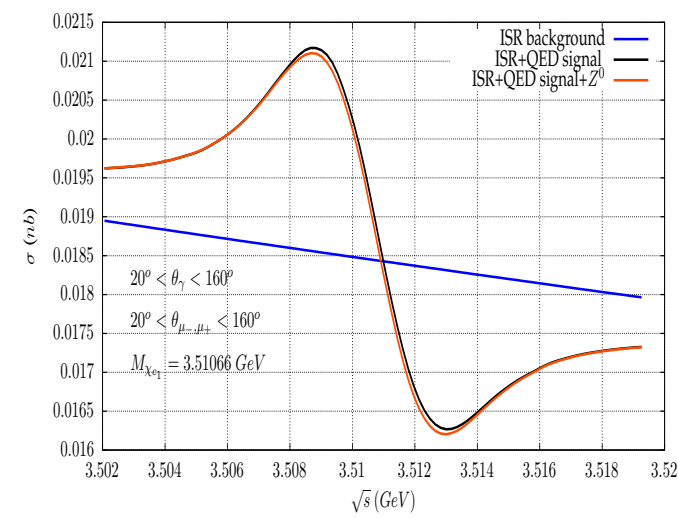

Figure 1. The cross section $e^{+} e^{-} \rightarrow \mu^{+} \mu^{-} \gamma$, see text for details.

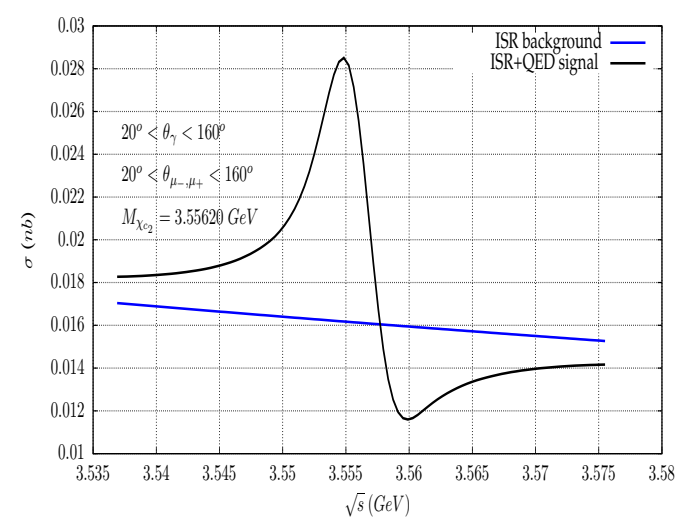

Figure 2. The cross section $e^{+} e^{-} \rightarrow \mu^{+} \mu^{-} \gamma$, see text for details.

is also model dependent. It can be tested experimentally with a scan experiment with energies chosen around the $\chi_{c_{1}}$ and $\chi_{c_{2}}$ masses.

The angular distributions of the muons are however more affected in regions, where the interference is not maximal. In Fig. 3 it is shown for $\chi_{c_{1}}$ and in Fig. 4 for $\chi_{c_{2}}$. The energy of the experiment in both cases was chosen to be equal to the mass of the $\chi_{c_{i}},(i=1,2)$ charmonium. In principle the studies of angular distributions would allow for detailed studies of the $\chi_{c_{i}}-J / \psi-\gamma$ amplitudes, but it would require collection of a big sample of events. Within the model used here [20] only one of the five allowed amplitudes gives contribution to the $\chi_{c_{2}}-J / \psi-\gamma$ amplitude and only one specific combination of the allowed amplitudes gives contribution to the $\chi_{c_{1}}-J / \psi-\gamma$ amplitude. Thus the predictions shown here are again specific to one of the models. Yet, one has to say that only in [20] the production of the $\chi_{c_{1}}$ and $\chi_{c_{2}}$ states in $e^{+} e^{-}$annihilation was studied, while other groups gave only predictions for the electronic widths. As the interference effects with the nonreducible background are crucial for these studies, the predictions of the electronic widths are not sufficient for the 


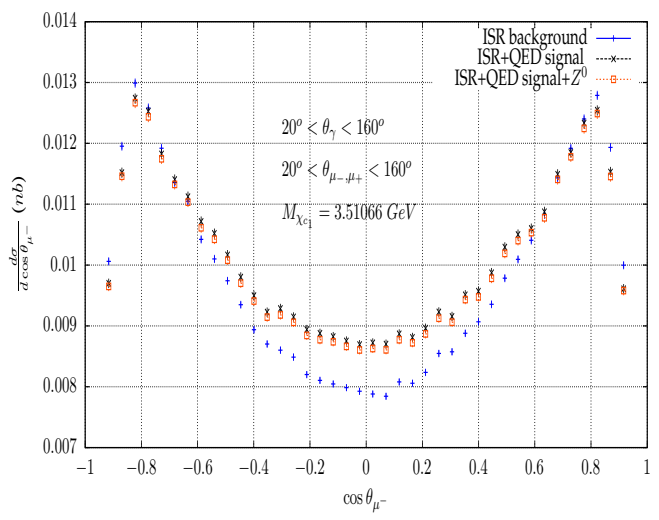

Figure 3. The differential (in $\mu^{-}$polar angle)cross section $e^{+} e^{-} \rightarrow \mu^{+} \mu^{-} \gamma$, see text for details.

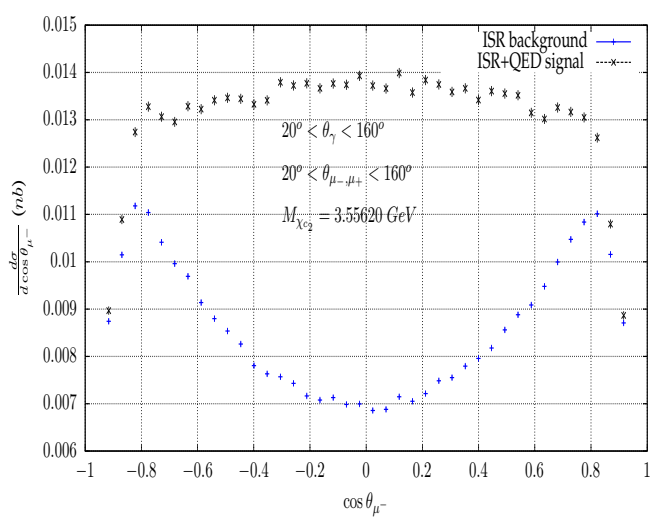

Figure 4. The differential (in $\mu^{-}$polar angle) cross section $e^{+} e^{-} \rightarrow \mu^{+} \mu^{-} \gamma$, see text for details.

extraction of the model parameters from the experimental data.

\section{EKHARA generator: $\chi_{c_{i}}, i=0,1,2$ production in $\gamma^{*}-\gamma^{*}$ processes}

It is clear from the previous section that a measurement of the $\chi_{c_{i}}-\gamma^{*}-\gamma^{*}$ form factors is crucial to test the models in detail. In [21] it was advocated that such measurement will be possible at BELLE2 experiment [30]. Here we report on this possibility showing also a complementary material not presented in [21]. The process which was advocated to be used is $e^{+} e^{-} \rightarrow e^{+} e^{-} \chi_{c_{i}}\left(\rightarrow J / \psi\left(\rightarrow \mu^{+} \mu^{-}\right) \gamma\right)$. Measurement of the muons and photon four-momenta will allow for a construction of the $\chi_{c_{i}}$ and $J / \psi$ invariant masses and thus for a clear identification of the final state. In the presented plots we assume that the invariant mass of the $\mu^{+} \mu^{-}$ pair is within ten $J / \psi$ widths from the mass of the $J / \psi$ and the invariant mass of the $\mu^{+} \mu^{-} \gamma$ particles is within ten $\chi_{c_{i}},(i=0,1,2)$ widths from its mass. We show the distributions of the event rates in the laboratory frame of the

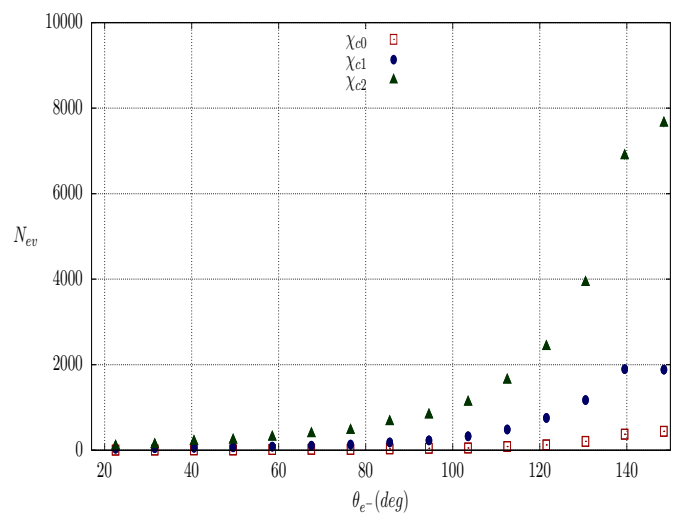

Figure 5. The differential (in $e^{-}$polar angle) cross section $e^{+} e^{-} \rightarrow e^{+} e^{-} J / \psi\left(\rightarrow \mu^{+} \mu^{-}\right) \gamma$, see text for details.

BELLE2 experiment assuming the asymmetric beams of 4 and $7 \mathrm{GeV}$ with the half crossing angle of $41.5 \mathrm{mrad}$. We assume also that the particles $\left(\mu^{+}, \mu^{-}\right.$, photon and electron or positron) can be detected and their four momenta measured if their polar angles are between $17^{\circ}$ and $150^{\circ}$ [31]. With the single tag events, when the final electron momenta is also measured, it will allow to cover the range of the $\chi_{c_{i}}-\gamma^{*}-\gamma^{*}$ transition form factor up to about $-25 \mathrm{GeV}^{2}$ for one of the invariants [21]. This corresponds to the polar angle distribution of the observed electron shown in Fig. 5. The number of events corresponds there to the integrated luminosity of $50 a b^{-1}$.

The accumulated luminosity of $50 a b^{-1}$ will allow also for a measurement of the $\chi_{c_{i}}-\gamma^{*}-\gamma^{*}$ transition form factors with both invariants substantially different from zero. In these case one has to measure both four momenta of the final electron and the final positron. The range of the $\gamma^{*}$ invariant masses which can be covered spans up to about $-10 \mathrm{GeV}^{2}$ [21]. It corresponds to the polar angles distribution of the final electron and final positron shown in Fig. 6 for $\chi_{c_{1}}$ and in Fig. 7 for $\chi_{c_{2}}$.

\section{Conclusions and near future developments}

In [20] a model of the $\chi_{c_{i}}-\gamma^{*}-\gamma^{*}$ and $\chi_{c_{i}}-J / \psi^{*}-$ $\gamma^{*}(i=1,2)$ transition form factors was developed. In [21] it was extended to cover also the case of $\chi_{c_{0}}$. It allowed [20] to predict the electronic widths of $\chi_{c_{1}}$ and $\chi_{c_{2}}$ and to show how to study the direct production of these states at BES-III experiment. In [21] it was shown that at BELLE2 experiment detailed studies of the $\chi_{c_{i}}-\gamma^{*}-\gamma^{*}$ transition form factors will be possible. The amplitudes predicted within the developed model were implemented into PHOKHARA9.2 (http://ific.uv.es/ rodrigo/phokhara/) and EKHARA2.2 (http://www.us.edu.pl/ ekhara) event generators. Hopefully the generators will serve well the experimental community, helping in performing the measurements discussed here. 


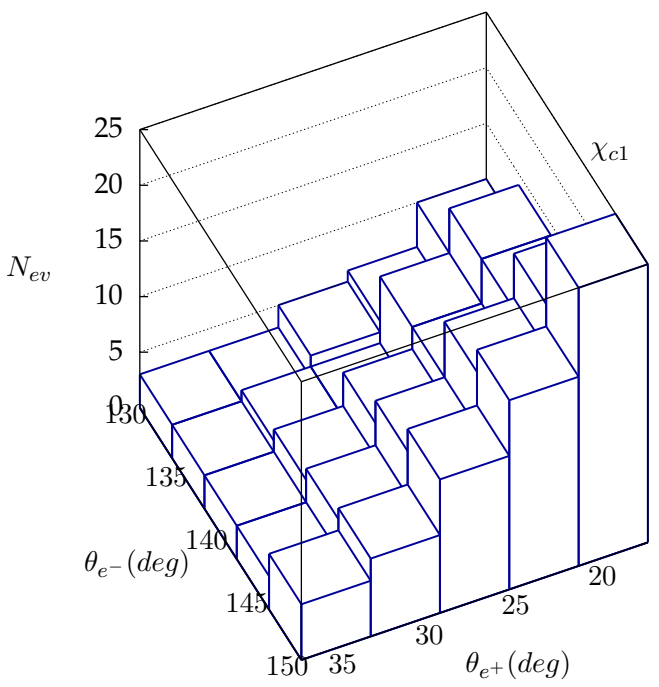

Figure 6. The differential (in $e^{-}$and $e^{+}$polar angles) cross section $e^{+} e^{-} \rightarrow e^{+} e^{-} \chi_{c_{1}}\left(\rightarrow J / \psi\left(\rightarrow \mu^{+} \mu^{-}\right) \gamma\right)$, see text for details.

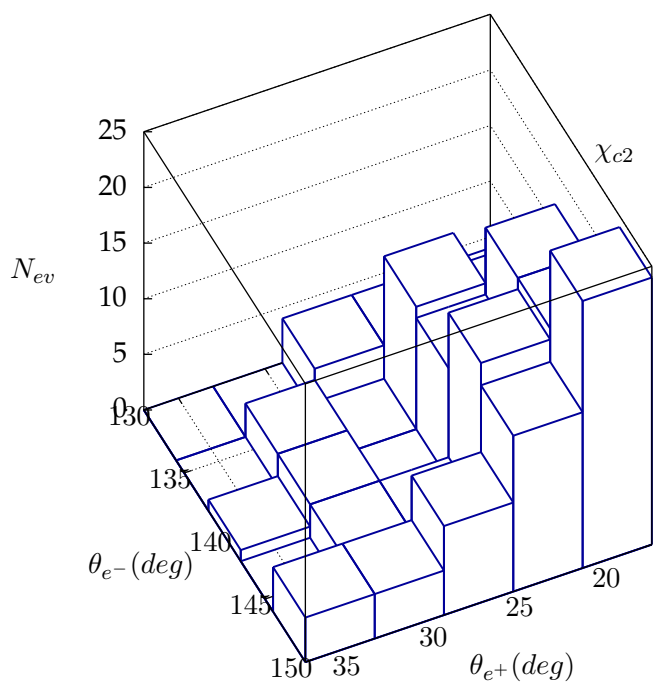

Figure 7. The differential (in $e^{-}$and $e^{+}$polar angles) cross section $e^{+} e^{-} \rightarrow e^{+} e^{-} \chi_{c_{2}}\left(\rightarrow J / \psi\left(\rightarrow \mu^{+} \mu^{-}\right) \gamma\right)$, see text for details.

The generators will be further extended in the near future: in PHOKHARA generator the radiative corrections for the reaction $e^{+} e^{-} \rightarrow \pi^{+} \pi^{-} \gamma$ will be completed, while in the EKHARA generator the radiative corrections will be included for the first time. Both works are well advanced.

\section{References}

[1] G. Rodrigo, H. Czyz, J.H. Kühn, M. Szopa, Eur.Phys.J. C24, 71 (2002), hep-ph/0112184

[2] H. Czyz, E. Nowak-Kubat, Phys. Lett. B634, 493 (2006), hep-ph/0601169
[3] G. Rodrigo, A. Gehrmann-De Ridder, M. Guilleaume, J.H. Kuhn, Eur. Phys. J. C22, 81 (2001), hep-ph/0106132

[4] H. Czyz, A. Grzelinska, J.H. Kuhn, G. Rodrigo, Eur. Phys. J. C27, 563 (2003), hep-ph/0212225

[5] H. Czyz, A. Grzelinska, J.H. Kuhn, G. Rodrigo, Eur. Phys. J. C33, 333 (2004), hep-ph/0308312

[6] H. Czyz, A. Grzelinska, J.H. Kuhn, Phys. Lett. B611, 116 (2005), hep-ph/0412239

[7] H. Czyz, A. Grzelinska, J.H. Kuhn, G. Rodrigo, Eur. Phys. J. C39, 411 (2005), hep-ph/0404078

[8] H. Czyz, A. Grzelinska, J.H. Kuhn, Phys. Rev. D81, 094014 (2010), 1002.0279

[9] H. Czyz, J.H. Kühn, S. Tracz, Phys. Rev. D90, 114021 (2014), 1407.7995

[10] H. Czyz, J.H. Kuhn, E. Nowak, G. Rodrigo, Eur. Phys. J. C35, 527 (2004), hep-ph/0403062

[11] H. Czyz, A. Grzelinska, J.H. Kuhn, G. Rodrigo, Eur. Phys. J. C47, 617 (2006), hep-ph/0512180

[12] H. Czyz, J.H. Kuhn, Eur. Phys. J. C18, 497 (2001), hep-ph/0008262

[13] H. Czyz, J.H. Kuhn, A. Wapienik, Phys. Rev. D77, 114005 (2008), 0804.0359

[14] H. Czyz, A. Grzelinska, J.H. Kuhn, Phys. Rev. D75, 074026 (2007), hep-ph/0702122

[15] F. Campanario, H. Czyż, J. Gluza, M. Gunia, T. Riemann, G. Rodrigo, V. Yundin, JHEP 02, 114 (2014), 1312.3610

[16] H. Czyz, J.H. Kuhn, Phys. Rev. D80, 034035 (2009), 0904.0515

[17] H. Czyż, M. Gunia, J.H. Kühn, JHEP 08, 110 (2013), 1306.1985

[18] H. Czyz, S. Ivashyn, Comput. Phys. Commun. 182, 1338 (2011), 1009.1881

[19] H. Czyz, S. Ivashyn, A. Korchin, O. Shekhovtsova, Phys. Rev. D85, 094010 (2012), 1202 . 1171

[20] H. Czyz, J.H. Kühn, S. Tracz, Phys. Rev. D94, 034033 (2016), 1605.06803

[21] H. Czyż, P. Kisza (2016), 1612.07509

[22] D.M. Asner et al., Int. J. Mod. Phys. A24, S1 (2009), 0809. 1869

[23] J.H. Kühn, J. Kaplan, E.G.O. Safiani, Nucl.Phys. B157, 125 (1979)

[24] R. Barbieri, R. Gatto, R. Kogerler, Phys. Lett. B60, 183 (1976)

[25] J. Kaplan, J.H. Kühn, Phys. Lett. B78, 252 (1978)

[26] D. Yang, S. Zhao, Eur. Phys. J. C72, 1996 (2012), 1203.3389

[27] N. Kivel, M. Vanderhaeghen, JHEP 02, 032 (2016), 1509.07375

[28] A. Denig, F.K. Guo, C. Hanhart, A.V. Nefediev, Phys. Lett. B736, 221 (2014), 1405. 3404

[29] C. Patrignani et al. (Particle Data Group), Chin. Phys. C40, 100001 (2016)

[30] T. Aushev et al. (2010), 1002. 5012

[31] T. Abe et al. (Belle-II) (2010), 1011.0352 